Supporting Information

\title{
Zwitterionic Polymer Micelles with Dual Conjugation of Doxorubicin and Curcumin: Synergistically Enhanced Efficacy against Multidrug-Resistant Tumor Cells
}

Guangfu Zhao, Yan Sun, and Xiaoyan Dong*

Department of Biochemical Engineering and Key Laboratory of Systems Bioengineering of the Ministry of Education, School of Chemical Engineering and Technology, Tianjin University, Tianjin 300072, China 
Table S1. Concentrations of doxorubicin (Dox) and curcumin (Cur) in Cur-pCB-Dox used in the uptake and efflux experiments. The concentrations of Dox and/or Cur in different formulations are also designed based on these two concentrations and listed in the table.

\begin{tabular}{ccc}
\hline Formulations & \multicolumn{2}{c}{ Concentration $(\mu \mathrm{g} / \mathrm{mL})$} \\
\cline { 2 - 3 } Cur-pCB-Dox & 50 & Cur \\
\hline pCB-Dox + pCB-Cur & 50 & 9.69 \\
pCB-Dox & 50 & 9.69 \\
pCB-Dox + Cur & 50 & - \\
pCB-Cur & 0 & 9.69 \\
Dox + pCB-Cur & 50 & 9.69 \\
Dox + Cur & 50 & 9.69 \\
Dox & 50 & 9.69 \\
Cur & - & - \\
\hline
\end{tabular}


Table S2. Properties of pCB-Dox, pCB-Cur and Cur-pCB-Dox conjugates

\begin{tabular}{cccccc}
\hline Conjugate & DS $^{\mathrm{a}}$ & Drug content $^{\mathrm{b}}$ & Zeta potential $^{\mathrm{c}}$ & Size $^{\mathrm{c}}$ & PDI \\
& $(-)$ & $(\mu \mathrm{g} / \mathrm{mL})$ & $(\mathrm{mV})$ & $(\mathrm{nm})$ & $(-)$ \\
\hline pCB-Dox & 3.10 & 48.6 & $24.64 \pm 2.55$ & $190.1 \pm 4.2$ & $0.573 \pm 0.031$ \\
pCB-Cur & 2.15 & 44.3 & $20.51 \pm 1.34$ & $91.3 \pm 5.9$ & $0.339 \pm 0.025$ \\
Cur-pCB-Dox & $1.10(\mathrm{Cur})$ & 11.7 (Cur) & $32.12 \pm 3.43$ & $164.2 \pm 4.8$ & $0.436 \pm 0.038$ \\
& 3.78 (Dox) & 64.2 (Dox) & & & \\
\hline
\end{tabular}

${ }^{a}$ Degree of substitution (DS) was defined as the number of conjugated molecules per one hundred CBMA repeating units in $\mathrm{pCB}$.

${ }^{\mathrm{b}}$ Data represent Dox and Cur concentrations for $\mathrm{pCB}-\mathrm{Dox}$ and $\mathrm{pCB}-\mathrm{Cur}$, respectively, and Dox and Cur concentrations for Cur-pCB-Dox as noted in the parentheses.

${ }^{c}$ Determined with DLS in $100 \mathrm{mM}$ phosphate buffer solution containing $10 \mathrm{mM}$ sodium chloride (pH7.4). 
Figure S1. Schematic for the syntheses of (a) CBMA and pCB, (b) pCB-SS-NH2, (c)
Dox-SS-COOH,
(d) pCB-Dox,
(e) Cur-SS-COOH,
(f) pCB-Cur and
(g)

Cur-pCB-Dox conjugates.

(a)

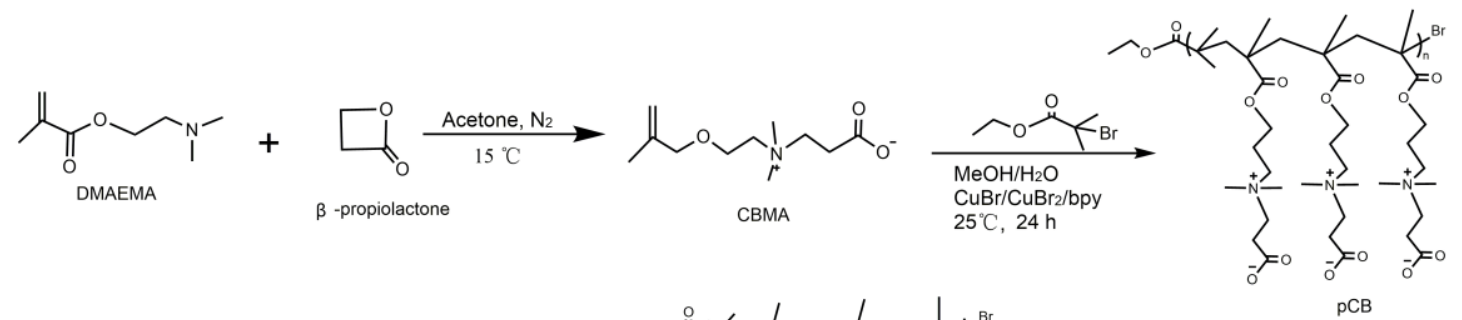

(b)

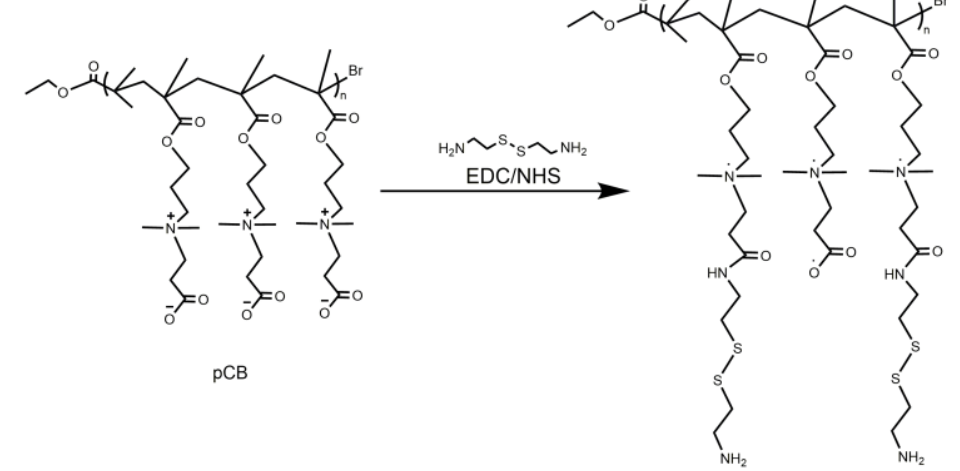

pCB-SS-NH2

(c)

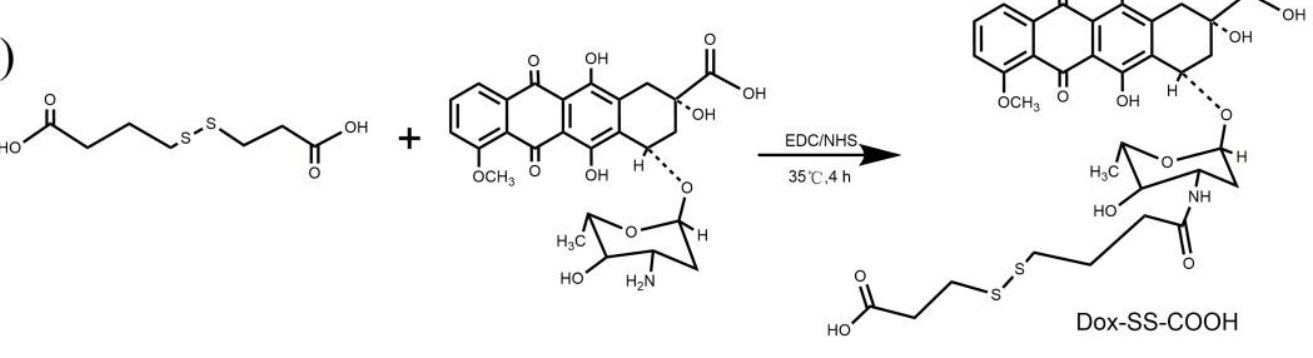

(d)
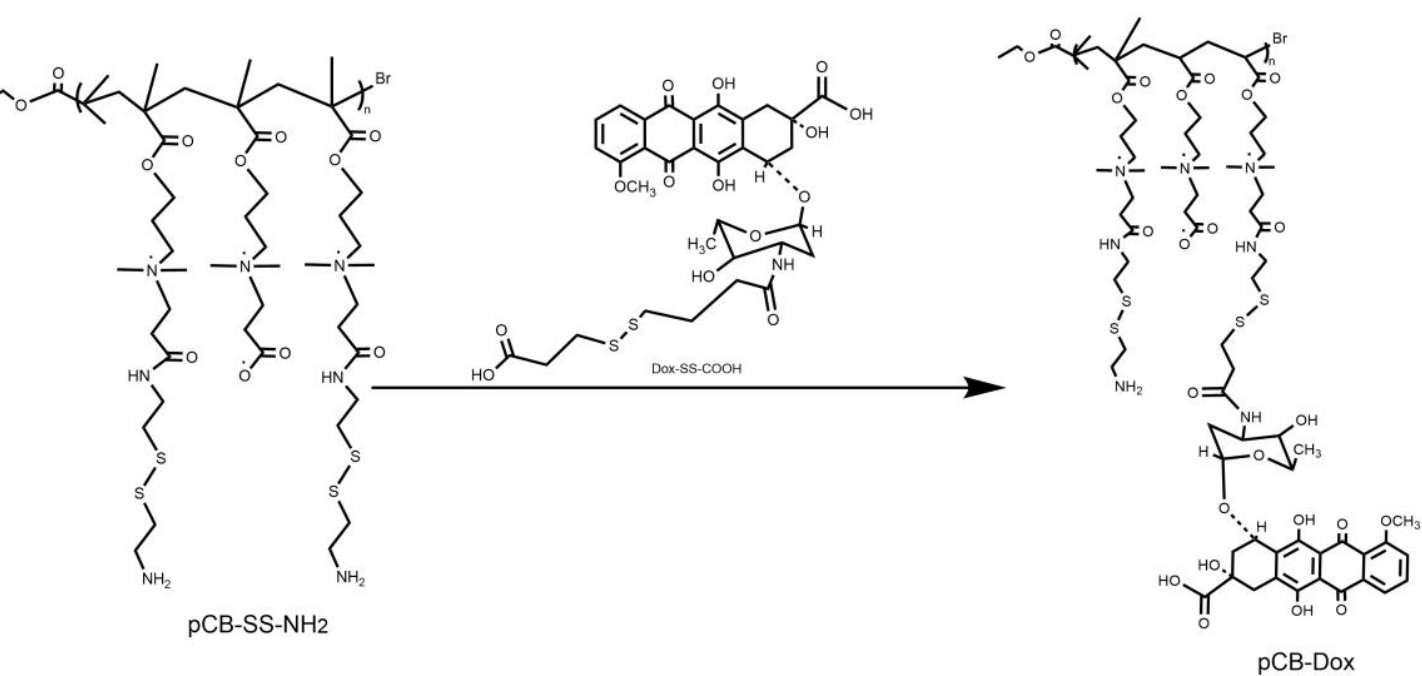


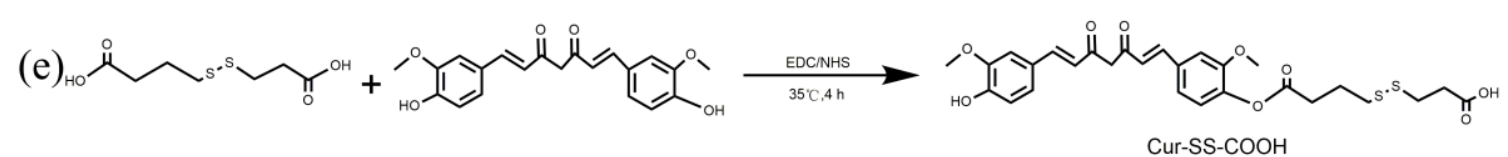

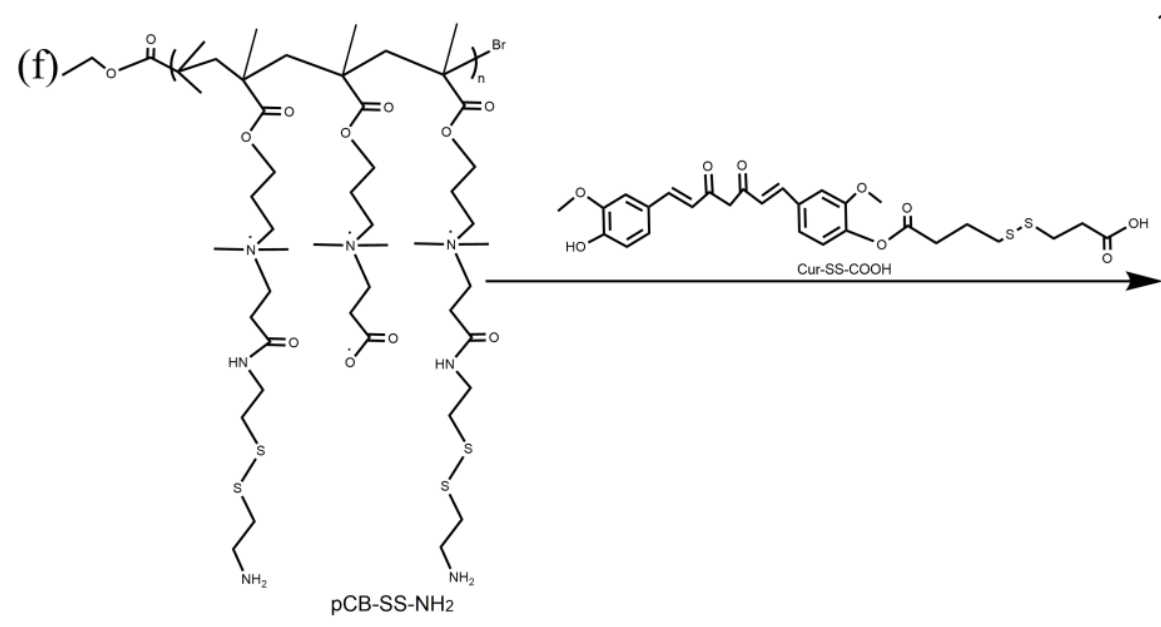
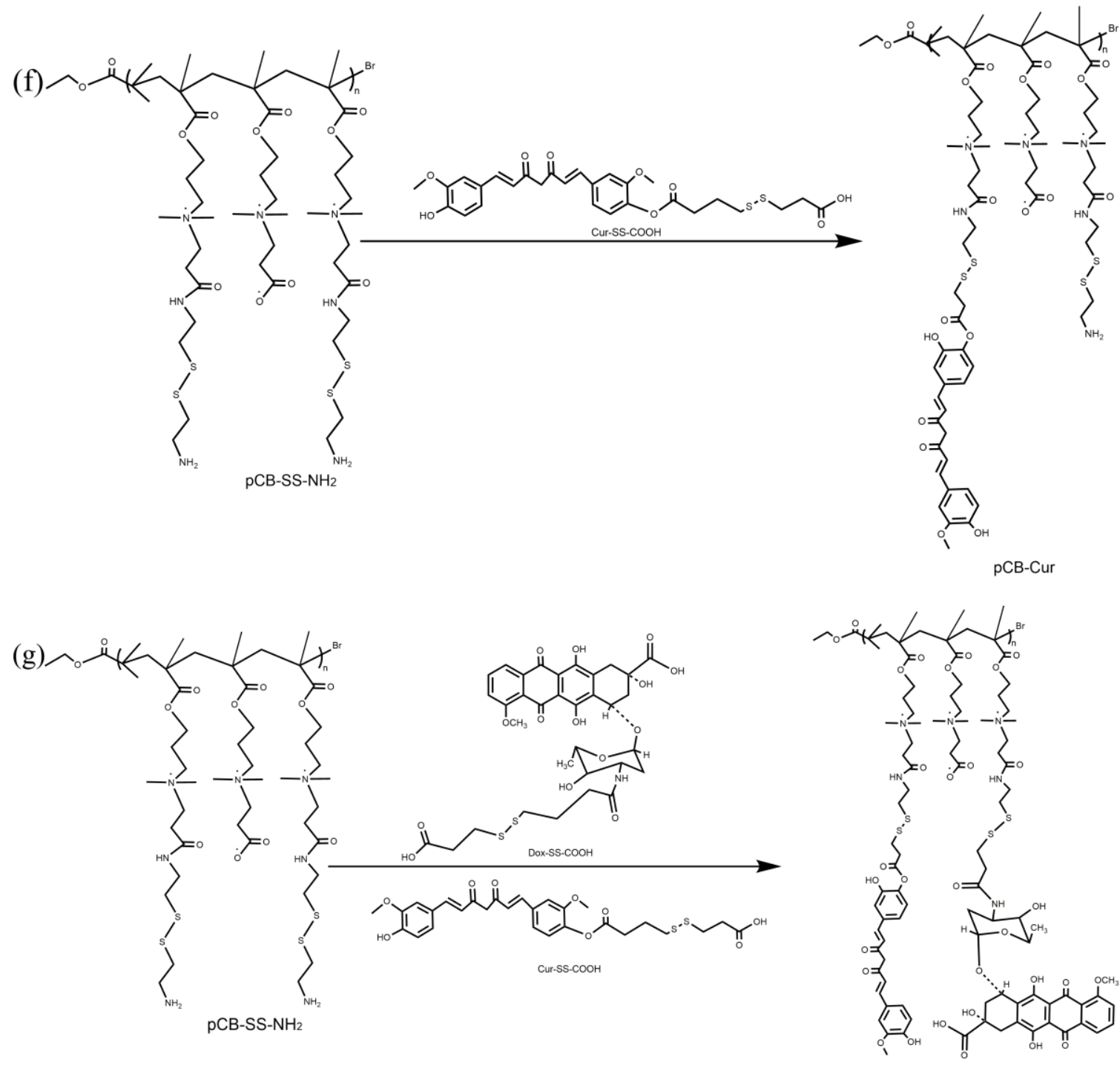

Cur-pCB-Dox 


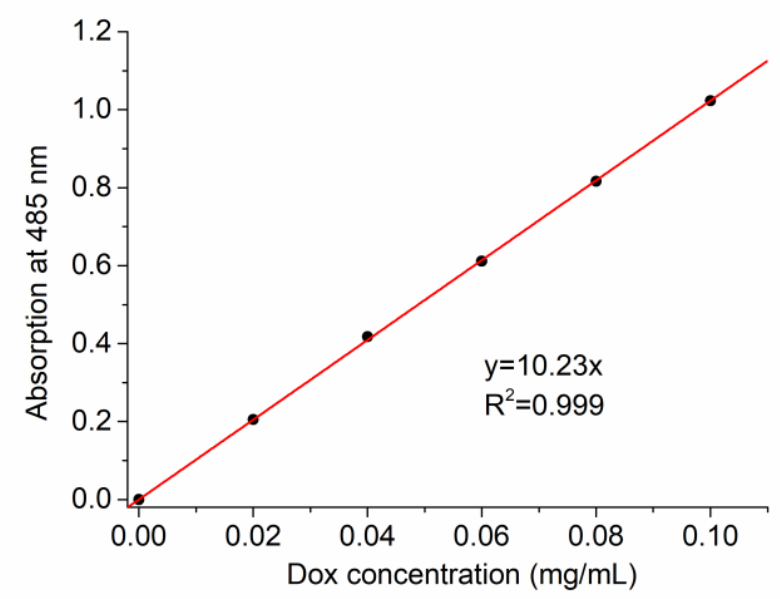

Figure S2. Calibration curve of Dox in water/DMSO (v/v=1:1). 


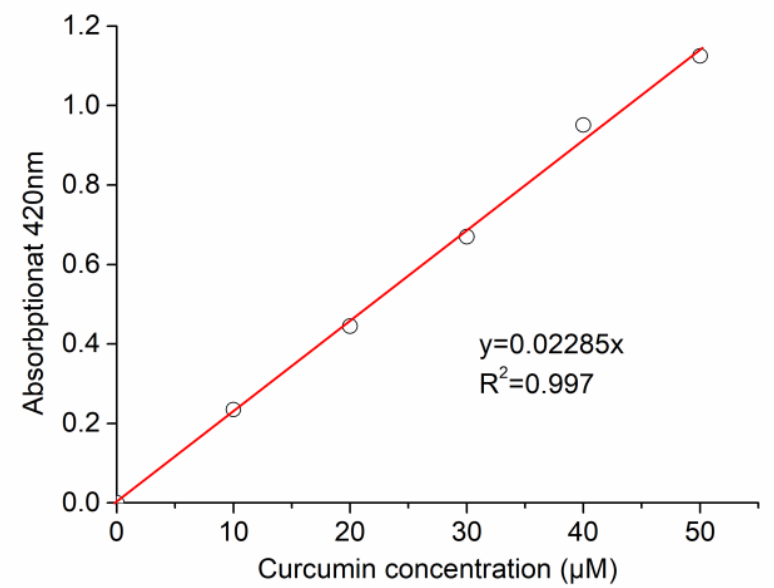

Figure S3. Calibration curve of Cur in water/DMSO (v/v=1:1). 

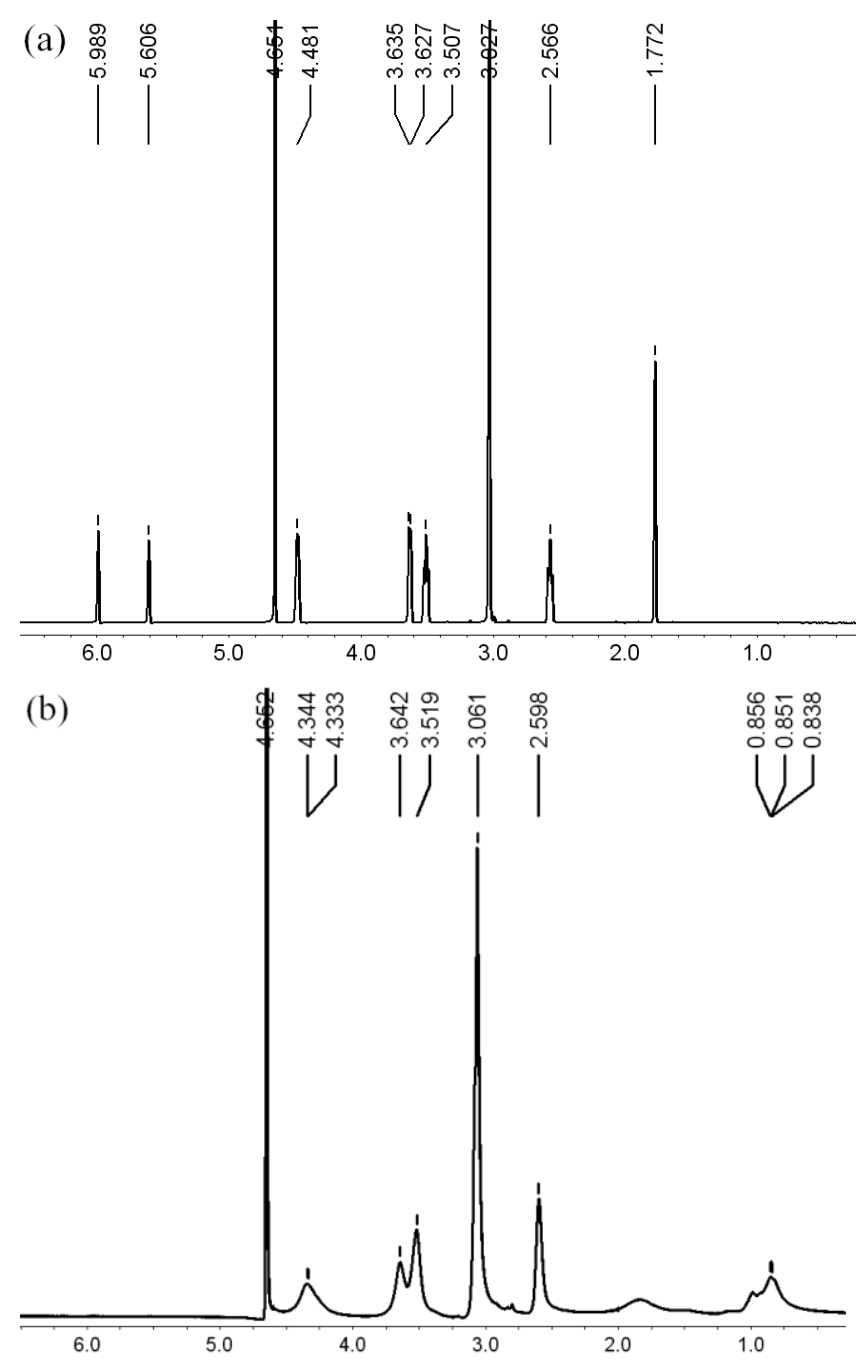

Figure S4. (a) NMR spectra of CBMA and (b) pCB in deuterated water. The ${ }^{1} \mathrm{H}$ NMR spectrum in Figure S4a. shows five typical peaks for the methyl groups of ethylenic bond at approximately $1.772 \mathrm{ppm}$, the methyl groups in nitrogen at $3.027 \mathrm{ppm}$, the ethyl groups in nitrogen at both 3.522 and $3.635 \mathrm{ppm}$, the ethyl groups linking with oxygen $\left(-\mathrm{O}-\mathrm{CH}_{2}-\right)$ at $4.481 \mathrm{ppm}$, and the proton groups of ethylenic bond at both 5.680 and 6.060 ppm. In comparison of Figures S4a and S4b, the peaks around 5.606 and $5.989 \mathrm{ppm}$ in the spectrum in Figure S4a are assigned to the protons of ethylenic bond, and the disappearance of the two peaks in the spectrum in Figure $\mathbf{S 4 b}$ shows the breaking of ethylenic bond. 


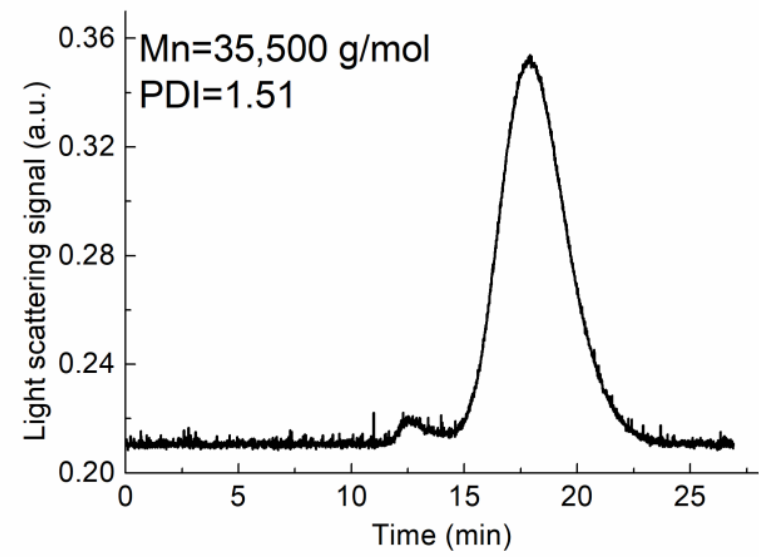

Figure S5. Gel permeation chromatography (GPC) profile of $\mathrm{pCB}$ with a mobile phase containing $0.1 \mu \mathrm{M} \mathrm{NaNO}_{3}$. 


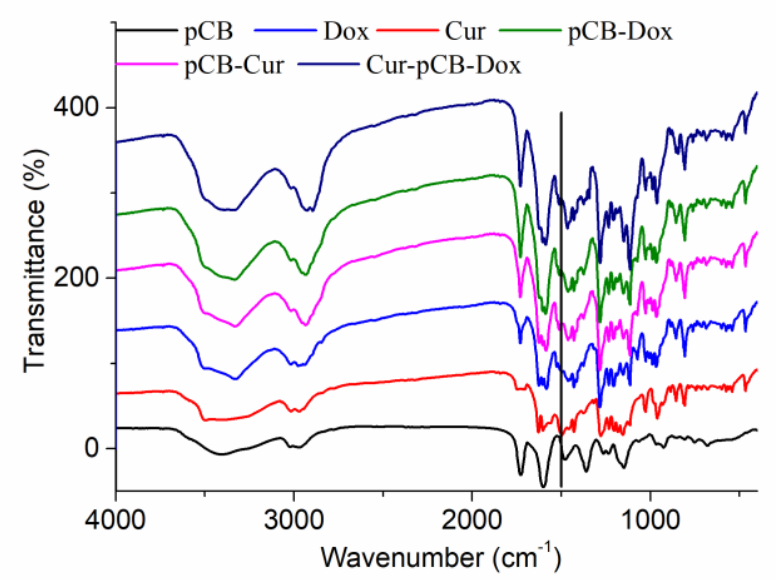

Figure S6. FT-IR spectra of pCB, Dox, Cur, pCB-Dox, pCB-Cur and Cur-pCB-Dox conjugates prepared by disulfide bond exchange methods.

In the FT-IR spectrum of $\mathrm{pCB}$, the broad band around $3500 \mathrm{~cm}^{-1}$ is attributed to the $\mathrm{O}-\mathrm{H}$ groups; the valley at $1602 \mathrm{~cm}^{-1}$ is associated with $\mathrm{C}-\mathrm{O}$ stretching of carboxylate anion. The FT-IR spectrum of pure Dox shows characteristic peaks at $3526 \mathrm{~cm}^{-1}$ due to $\mathrm{N}-\mathrm{H}$ stretching vibration for primary amine structure and at $3330 \mathrm{~cm}^{-1}$ due to $\mathrm{O}-\mathrm{H}$ stretching vibration; also, the $\mathrm{N}-\mathrm{H}$ bending is observed in the range of 1617 and 1580 $\mathrm{cm}^{-1}$. The peak at $3414.71 \mathrm{~cm}^{-1}$ is found in the spectrum of Cur for phenolic hydroxyl group, and the $\mathrm{C}=\mathrm{C}$ band of benzene with a stronger absorption peak at $1510 \mathrm{~cm}^{-1}$ is covered in the spectrum of Cur, which confirms the successful synthesis of pCB-Cur and Cur-pCB-Dox. 


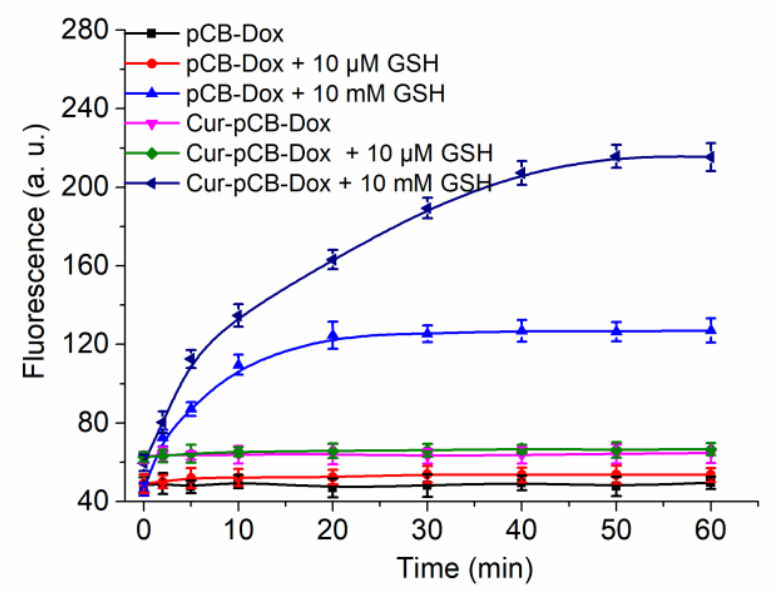

Figure S7. Release kinetics of Dox in pCB-Dox and Cur-pCB-Dox conjugates in the presence or absence of glutathione (GSH), determined by a fluorescence spectrophotometer (Perkin Elmer LS-55, MA, USA). 


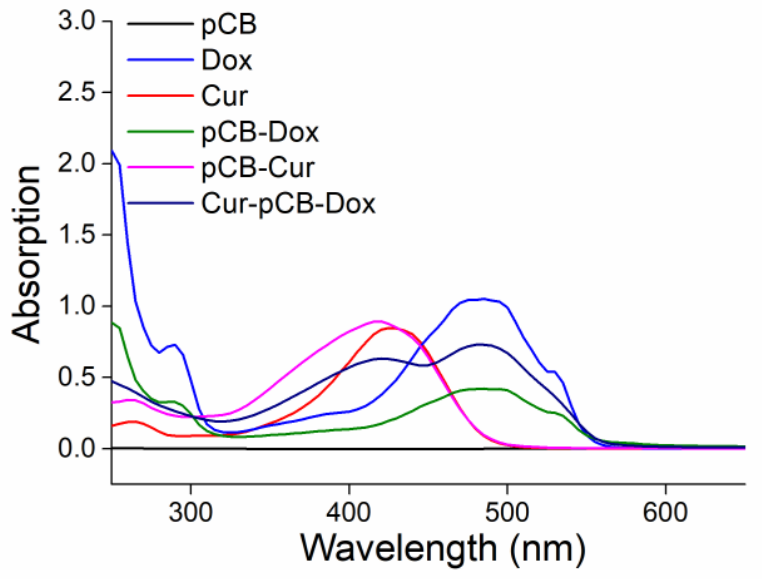

Figure S8. UV-vis spectra of pCB, Dox, Cur, pCB-Dox, pCB-Cur and Cur-pCB-Dox conjugates. 

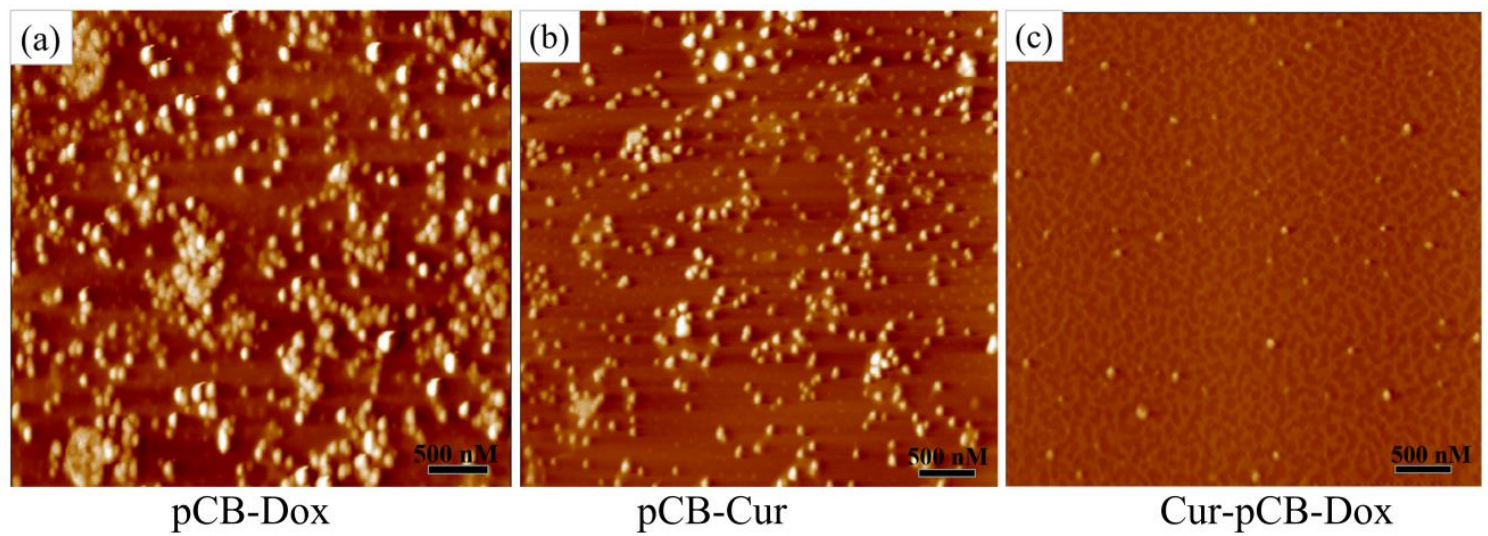

Figure S9. AFM images of (a) pCB-Dox, (b) pCB-Cur and (c) Cur-pCB-Dox. 


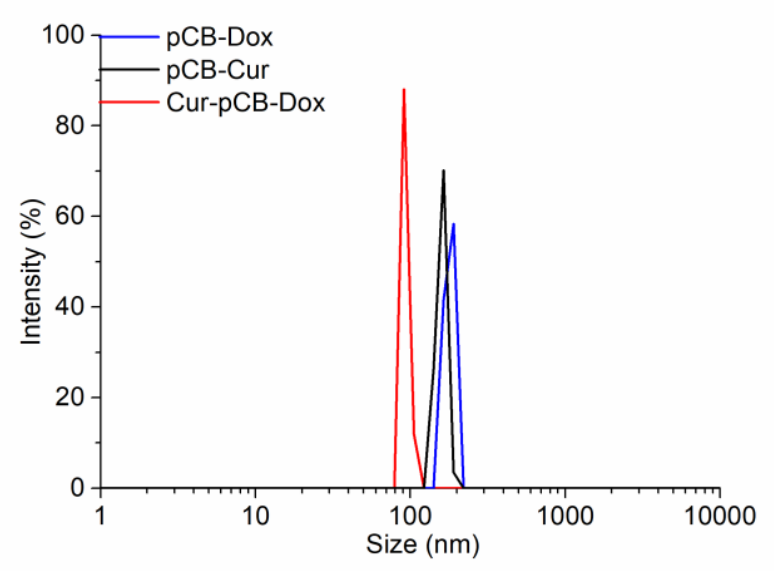

Figure S10. Size distributions of self-assembled pCB-Dox, pCB-Cur and Cur-pCB-Dox in PBS (100 mM phosphate buffer solution with $10 \mathrm{mM}$ sodium chloride, $\mathrm{pH} 7.4)$. 

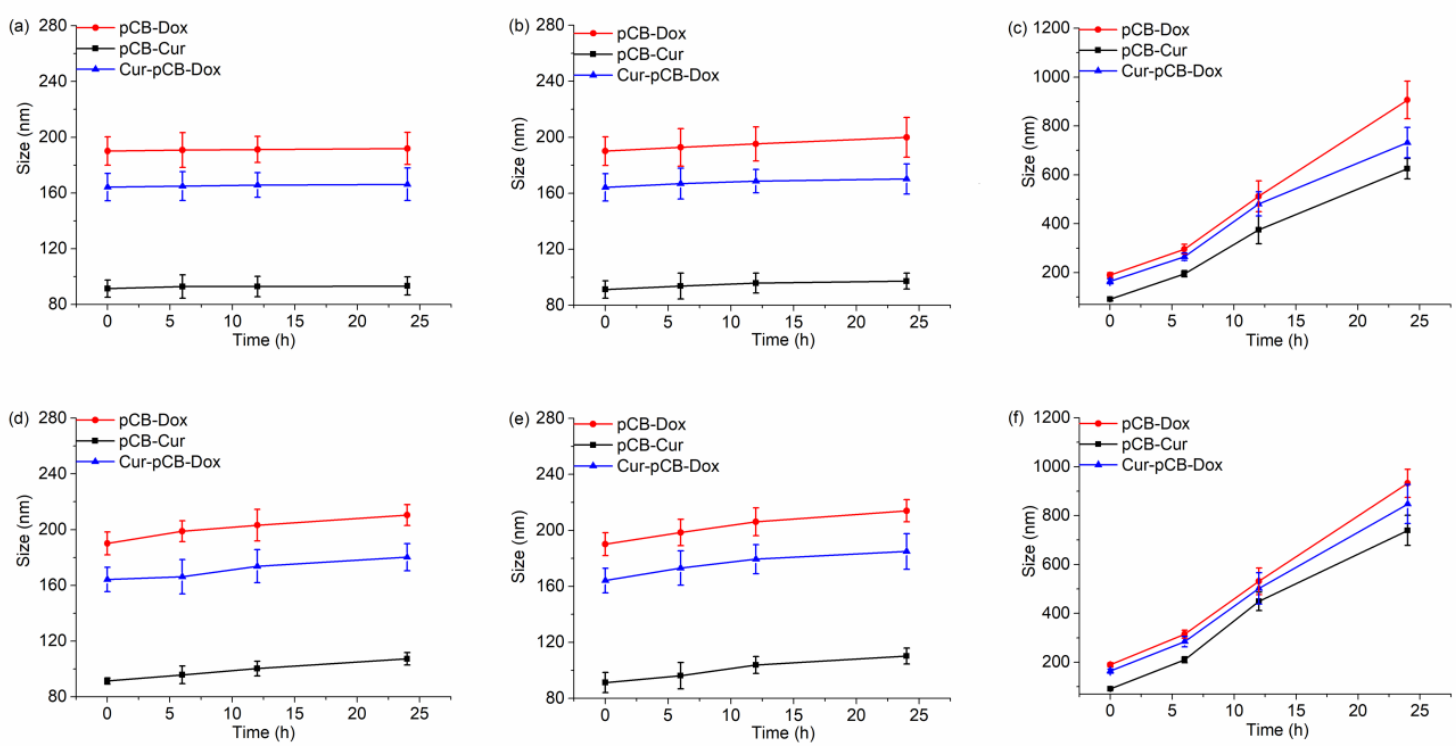

Figure S11. Redox-induced size changes of pCB-Dox, pCB-Cur and Cur-pCB-Dox with incubation time followed by DLS measurements under different conditions. (a) pH 7.4 + $0 \mu \mathrm{M}$ GSH, (b) pH 7.4 + $10 \mu \mathrm{M}$ GSH, (c) pH 7.4 +10 mM GSH, (d) pH 5.0 $+0 \mu \mathrm{M}$ GSH, (b) $\mathrm{pH} 5.0+10 \mu \mathrm{M}$ GSH and (c) pH 5.0 +10 mM GSH. 

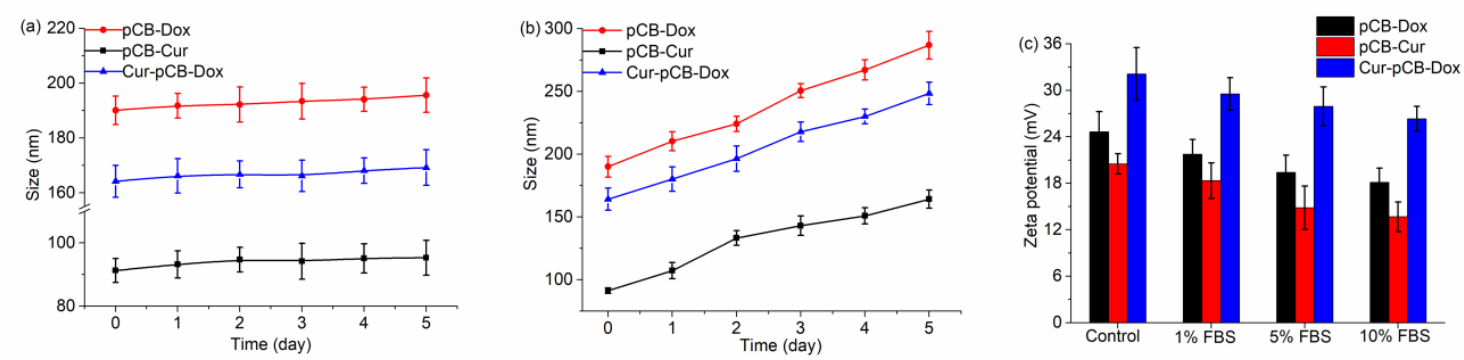

Figure S12. The colloidal stability, described by (a and b) size and (c) zeta potential described by size changes, of pCB-Dox, pCB-Cur and Cur-pCB-Dox (a) Size changes in $10 \%$ FBS solution during the 5-day incubation at $\mathrm{pH} 7.4$ (b) Size changes in $10 \%$ FBS solution during the 5-day incubation at $\mathrm{pH} 5.0$ (c) Zeta potential changes in 1\%, $5 \%$ and $10 \%$ FBS solutions at $37^{\circ} \mathrm{C}$ for $24 \mathrm{~h}$. 

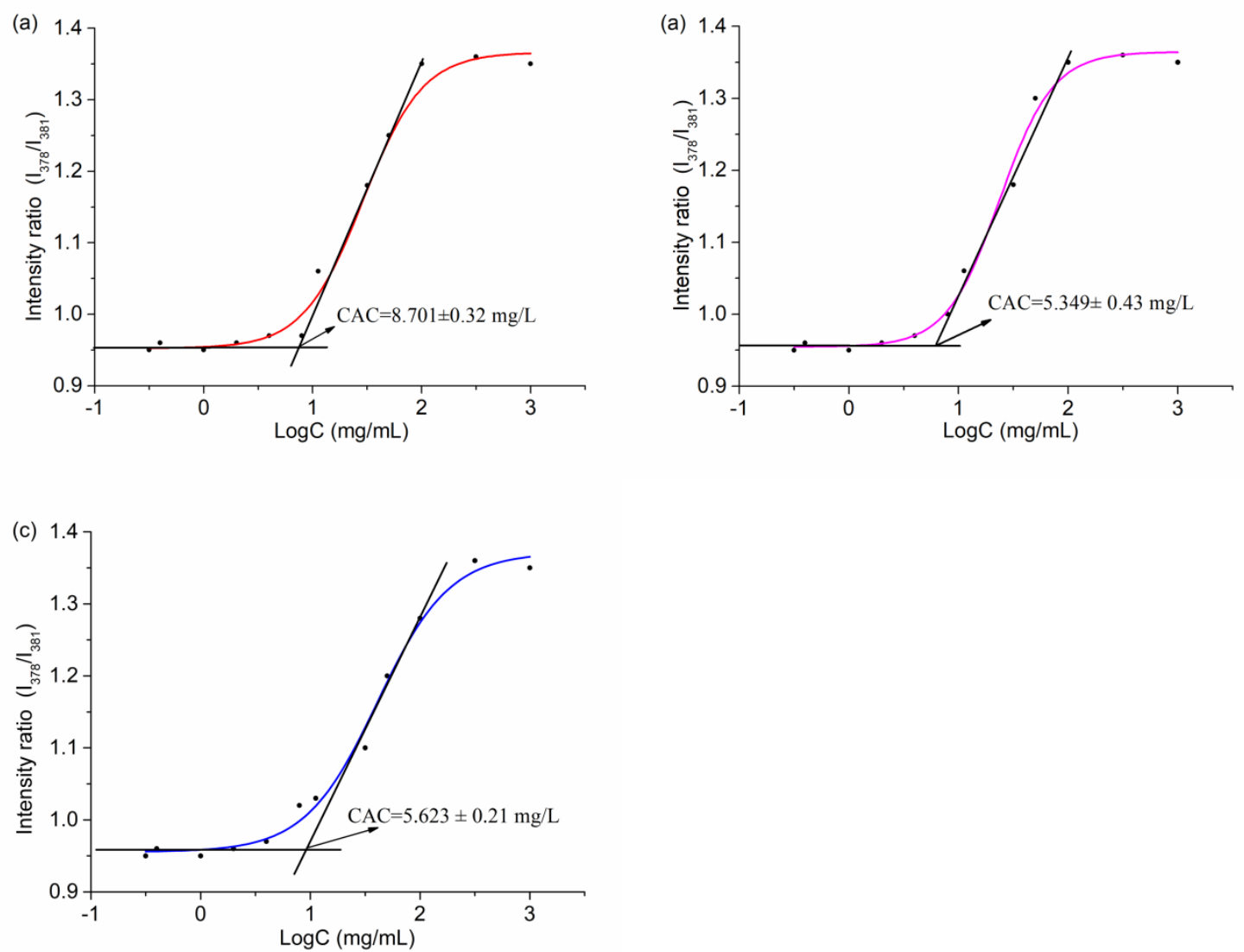

Figure S13. Critical aggregation concentration (CAC) measurement using pyrene as the fluorescent probe: plot of intensity ratio between $I_{378} / I_{381}$ as a function of concentration in PBS. (a) pCB-Dox, (b) pCB-Cur and (c) Cur-pCB-Dox. 

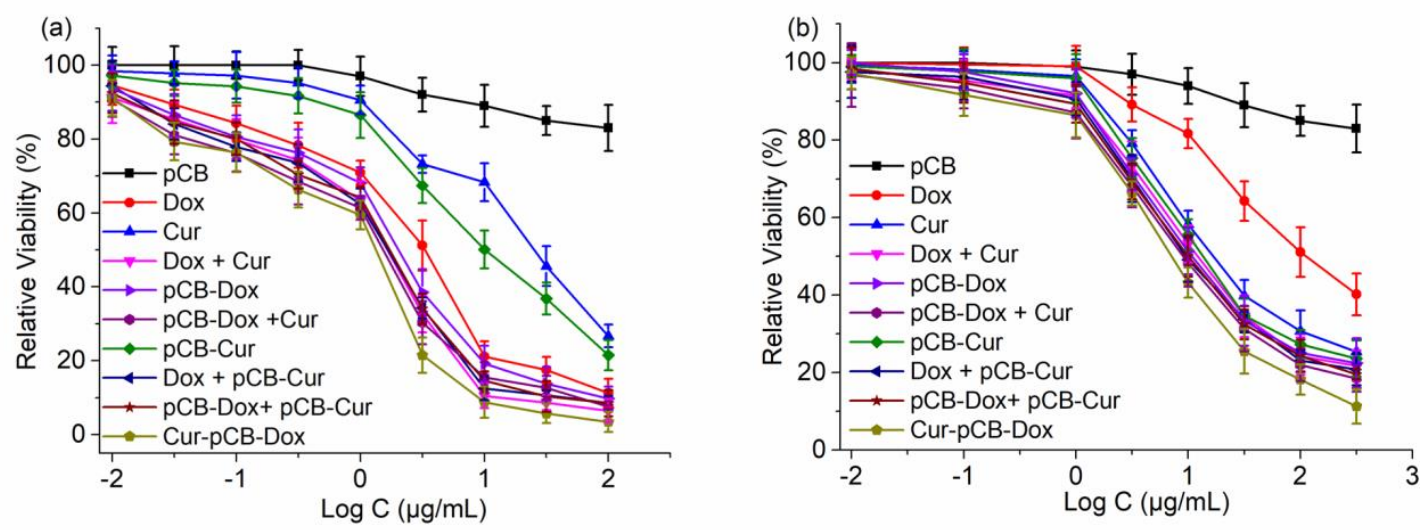

Figure S14. In vitro cytotoxicity of different chemical agents against (a) MCF-7 and (b) MCF-7/Adr cells after incubation for $48 \mathrm{~h}$ at $37^{\circ} \mathrm{C}$ (mean $\left.\pm \mathrm{SD}, n=3\right)$. Cells $(5 \times$ $10^{3}$ cells/well) were cultured in RPMI-1640 medium containing one of the agents/formulations (pCB, Dox, Cur, Dox + Cur, pCB-Dox, pCB-Dox + Cur, pCB-Cur, Dox + pCB-Cur, pCB-Dox + pCB-Cur and Cur-pCB-Dox) for $48 \mathrm{~h}$. The cells incubated with medium only were used as control. The $x$ axis, $C$, represents Dox or Cur concentration for the single agent systems (Dox/pCB-Dox or Cur/pCB-Cur), respectively. For the formulations containing both Dox and Cur (i.e., Dox + Cur, pCB-Dox + Cur, Dox + pCB-Cur, pCB-Dox + pCB-Cur and Cur-pCB-Dox $), C$ refers to Dox concentration, and the concentration ratio of Dox/Cur used in the cytotoxicity is 5.16 (the same as in Cur-pCB-Dox, see Table S1). For the pCB group, $C$ refers to pCB concentration which covers the $\mathrm{pCB}$ concentration range used in the Cur-pCB-Dox conjugate group. 